\title{
Qualitätskontrolle und Transparenz: Langfristig zählen nur konkrete Aktionen
}

\author{
Pietro Regazzoni, \\ Alberto Fernandez, \\ Peter Kleist, \\ Stephan M. Perren \\ * Die Literaturangaben finden \\ sich unter www.saez.ch \\ $\rightarrow$ Aktuelle Nummer oder \\ $\rightarrow$ Archiv $\rightarrow 2013 \rightarrow 29 / 30$.
}

\begin{abstract}
Die heutige gesundheitspolitische Diskussion dreht sich häufig um Begriffe wie «Massnahmen zur Qualitätssicherung» und «Förderung einer hochstehenden klinischen Forschung». Zweifellos handelt es sich dabei um wesentliche Voraussetzungen für Fortschritte in der medizinischen Versorgung. Entscheidend sind dabei aber Taten, nicht Schlagworte. Die FMH hat am 27.11.2012 die Schweizerische Akademie für Qualität in der Medizin gegründet und versucht damit, die Qualitätskontrolle in der Hand der Ärzte zu behalten. Solche Vorstösse von medizinischer Seite sind zu begrüssen und könnten mithelfen, dem laufenden «Verlust an Entscheidungskompetenz» der Ärzte entgegenzuwirken [1-3] ${ }^{*}$, und allenfalls auch dazu beitragen, die vieldiskutierte «Surconsommation Médicale» (bis hin zu angeblich unnötigen Diagnose- und Therapiemassnahmen mit ihren Kostenfolgen) in den Griff zu bekommen [4].
\end{abstract}

\section{Komplikationen und Probleme sind in der Medizin system- inhärent, können Lerneffekte und Prozessänderungen generieren und eine Quelle des Fortschritts sein.}

Korrespondenz:

Prof. Dr. med. Pietro Regazzoni Sentiero per i Ciapei 8 Strada di Soragno CH-6964 Lugano-Soragno

p_regazzoni[at]bluewin.ch
Einen ersten Schritt im Sinne von Transparenz zur Qualitätskontrolle hat die »Society of Cardiothoracic Surgery of Great Britain and Ireland» getan, indem sie seit 2005 die Mortalitätsraten für definierte Eingriffe in verschiedenen Spitälern und durch verschiedene Chirurgen publiziert [5]. Die Aussagekraft solcher Daten setzt jedoch voraus, dass auch die Schwere der Fälle einbezogen wird. Die Angst vor dem Verlust der Entscheidungskompetenz [2, 3] wurde neutralisiert durch das Einbinden der Mitglieder während der gesamten Untersuchung (Auswahl der Erfolgskriterien, Vorgehen bei fehlenden Daten, Information der Studienteilnehmer und Spitäler usw). Die Glaubwürdigkeit der Daten wurde dadurch erhöht, dass für die externe Prüfung (neutrales Auditing) nicht nur die Mortalitätsdaten zur Verfügung stehen mussten, sondern auch die Prozesse offenzulegen waren, mit denen die Daten erhoben und analysiert wurden.

In die gleiche Richtung geht - im Sinne von Transparenz bei der Publikation wissenschaftlicher Daten - die Geste von GlaxoSmithKline (GSK): Der Cochrane Collaboration wurden die vollständigen,

\section{Contrôle de la qualité et transpa- rence: à long terme, seules les actions concrètes comptent}

La crédibilité des publications scientifiques peut être améliorée si les données brutes obtenues lors des études sont toutes, sans exception, facilement accessibles et disponibles en vue d'un contrôle (auditing) ultérieur neutre. En la matière, l'application au domaine médical de ce qui se fait déjà dans l'aviation devrait indirectement induire une baisse des coûts globaux de la santé. Mais pour cela, il faut un soutien politique clair. De facto, une documentation détaillée des interventions chirurgicales - qui tiendrait également compte des difficultés et des incidents (à l'instar d'une «boîte noire» chirurgicale) pourrait devenir un précieux outil d'apprentissage. Mais il faudra impérativement garantir l'anonymat des données et procéder à certaines réformes de la législation sur la responsabilité civile pour s'assurer la participation des médecins et éviter une médecine défensive qui ne ferait que nuire aux patients.

anonymisierten Daten von 30 klinischen Studien überlassen. Zwar konstatieren die Cochrane Reviewer Probleme in Bezug auf Analyse und Interpretation der Daten [5], ein erster wichtiger Schritt ist aber getan. Der Medizinische Direktor von GSK in der Schweiz fordert in einem vor kurzem in der Schweizerischen Ärztezeitung veröffentlichten Artikel vier konkrete Schritte zu mehr Transparenz [6]:

1. Die Verpflichtung zur Registrierung aller klinischer Studien in einem öffentlich zugänglichen Register

2 die Veröffentlichung der Studienprotokolle;

3. die Bereitstellung der Studienberichte einschliesslich der Rohdaten bis auf Ebene individueller Studienteilnehmer und

4. die Verpflichtung zur Veröffentlichung aller klinischer Studien. 


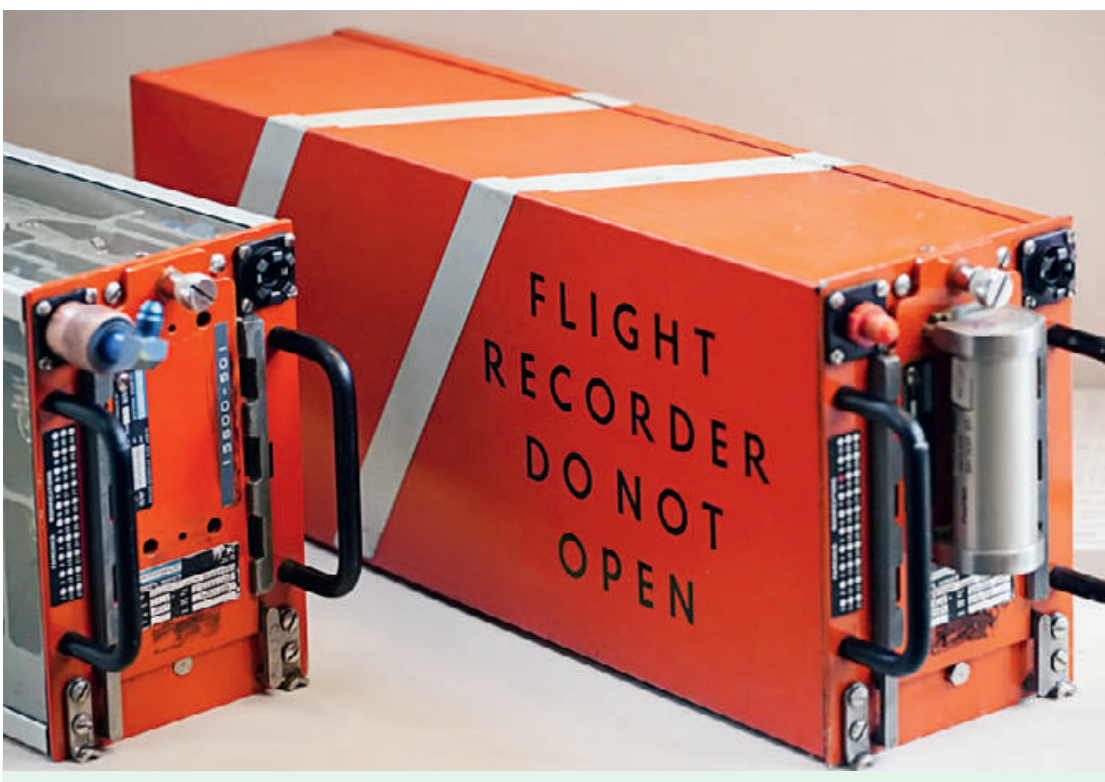

Vorbild Aviatik: Ein Flugschreiber zeichnet alle relevanten Daten auf. Dies sollte bei Operationen ebenfalls so sein.
Schwierigkeiten und Komplikationen ist eine notwendige Voraussetzung, um eine derartige Dokumentation auch in der Chirurgie didaktisch zu verwerten. Mit Ausnahme gewisser endoskopischer Eingriffe fehlt jedoch eine solche Dokumentation bei Operationen, obschon die technischen Voraussetzungen dafür vorhanden sind.

Komplikationen und Probleme sind in der Medizin systeminhärent, können auch hier Lerneffekte und Prozessänderungen generieren und damit eine wertvolle Quelle für den Fortschritt darstellen. Nur wenige Komplikationen sind Folge eines schuldhaften Fehlers. Komplikationen sind aber auch eine wichtige Ursache von Gesundheitskosten. Die Vermeidung von Komplikationen bzw. die Kenntnis, unter welchen Umständen sie gehäuft auftreten, tragen dazu bei, die globalen Gesundheitskosten zu senken. Die globalen Gesundheitskosten lassen sich weiter senken, wenn dem Druck betriebswirtschaftlicher Überlegungen der Spitaldirektionen widerstanden wird. Die Umsetzung von Erfahrungen der Aviatik in die Medizin ist nicht einfach. Die Zurückhaltung der Ärzte entspringt wohl der begründeten Befürchtung vor rechtlichen Konsequenzen (Haftpflicht).

Eine garantierte Anonymisierung kann die Befürchtungen mindern und die Umsetzung dieser Vorschläge fördern, zumal die Angst vor juristischen Folgen eine defensive Haltung bei den Medizinern bewirkt. Sie schaden dadurch jenen Patienten, für die beispielsweise ein schwieriger und risikoreicher, aber dringend notwendiger Eingriff lebensrettend sein kann [9-14].

\section{Zusammenfassung}

Die Glaubwürdigkeit wissenschaftlicher Publikationen wird erhöht, wenn ausnahmslos alle, während einer Studie anfallenden, Rohdaten einfach einsehbar sind und für eine neutrale, nachträgliche Kontrolle («Auditing») zur Verfügung stehen. Die Umsetzung der aufgelisteten Vorschläge in der Medizin und die erwartete Senkung der Globalkosten bedingt eine klare politische Unterstützung.

Eine detaillierte Dokumentation chirurgischer Eingriffe - unter Einschluss von Schwierigkeiten und Zwischenfällen - («chirurgische Black Box») könnte ein wertvolles Lernwerkzeug werden.

Eine garantierte Anonymisierung sowie gewisse Reformen der Haftpflichtgesetzgebung sind unabdingbar, um die notwendige Kooperation der Ärzte zu garantieren und eine für die Patienten nachteilige defensive Medizin zu vermeiden. (continuous) mit qualitativ hochstehenden Bildern (ICUC) dokumentieren [8]. Der Einschluss allfälliger 


\section{Literatur}

1 Bauer W. Deprofessionalisierung - ein nüchternes Wort für eine unerfreuliche Diagnose. Schweiz Ärztezeitung. 2010;91(5):196.

2 Unschuld P. Der Arzt als Fremdling in der Medizin. München: Zuckschwerdt Verlag; 2005.

3 Unschuld P. Ware Gesundheit. München: C. H. Beck Verlag; 2009.

4 Schäfer F. Chirurgen warnen vor unnötigen Operationen. Berner Zeitung. 7.3.2013.

5 Godlee F. Promises of Transparency? Hold the applause. BMJ. 2013;346:f1513.

6 Kleist P. Vier Schritte zu mehr Transparenz in der klinischen Forschung. Schweiz Ärztezeitung. 2013; 94(12):483-5

7 Staender S, Davies J, Helmreich B, et al. The anaesthesia critical incidence reporting system. Int. J Med Inform 1997; 47: 87-90.

8 ICUC iBooks: www.ICUC.ch
9 Anderson RE. Billions for defense: the pervasive nature of defensive medicine. Arch Intern Med. 1999;159:2399-402.

10 Brennan TA, Mello MM, Studdert DM. Liability, Patient Safety, and Defensive Medicine. In: Sage WM, Kersh R (Eds.): Medical malpractice and the U.S. health care system. 2006; S. 112.

11 Chandra A et al. Defensive Medicine may be costlier than it seems. Wall Street Journal. Feb 7th 2013.

12 DeKay ML, Asch DA. Is the defensive use of diagnostic tests good for patients, or bad? Med Decis Making. 1998;18:19-28.

13 Panting G. Doctors on the defensive. The Guardian (London). April 1st 2005.

14 Studdert DM, Mello MM, Sage WM, et al. Defensive medicine among high-risk specialist physicians in a volatile malpractice environment. JAMA. 2005;293:2609-17. 